\title{
Author Rebuttal to Rocha et al. "Comments on Minimizing Buffer Requirements under Rate-Optimal Schedule in Regular Dataflow Networks"
}

\author{
R. Govindarajan • Guang R. Gao
}

Published online: 14 April 2015

(c) Springer Science+Business Media New York 2015

The claims made in our paper (Govindarajan, et al., JVLSI 2002) are indeed correct. For the example discussed in page 212 of our paper (reproduced here in Fig. 1) and the schedule given in page 211 (reproduced here in Table 1), the buffer requirements for all arcs are shown in Table 2.

It can be seen that the minimum buffer requirements for $\operatorname{arcs}(a, b),(b, a),(b, d),(d, b),(c, d)$, and $(d, c)$, are respectively $2,3,2,3,6$, and 6 . And the total buffer requirement is indeed 22. It is possible to have other rate-optimal schedules which may have a buffer requirement which is more than the minimum requirement.

As stated in our paper, the buffer requirement is ONLY during the steady-state execution of a minimum buffer rateoptimal schedule. The reasons for following this approach, as stated in our paper, is that the steady-state kernel is what is executed repeatedly (significantly more number of times

\section{R. Govindarajan $(\bowtie)$}

Supercomputer Education and Research Center, Indian Institute of Science, Bangalore 560 012, India

e-mail: govind@serc.iisc.ernet.in

G. R. Gao

Department of Electrical and Computer Engineering,

University of Delaware, Newark, DE 19716, USA

e-mail: ggao.capsl@gmail.com

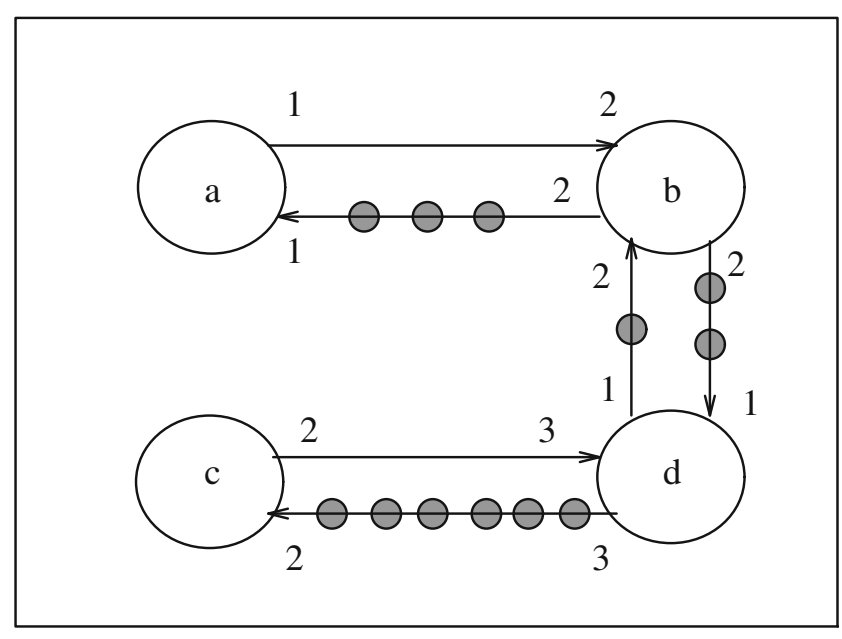

Figure 1 Example RSFG.

than the prologue or epilogue) and hence optimizing the kernel and its buffer requirement is beneficial, even if it requires additional resources (compile time).

With respect to the arguments in page 3 (of the submitted note by Rocha, et al.,) and Equations (9)-(11), our comment is that the invariant calculation is possibly giving the maximum buffer requirement for the individual places and it is not clear how the maximum buffer requirement invariant for a group of places correspond to the minimum buffer requirement of a rate-optimal schedule. 
Table 1 Schedule for the example RSFG.

\begin{tabular}{|c|c|c|c|c|c|c|c|c|c|c|c|c|c|}
\hline & \multicolumn{13}{|c|}{ Time step } \\
\hline & 0 & 1 & 2 & 3 & 4 & 5 & 6 & 7 & 8 & 9 & 10 & 11 & 12 \\
\hline iteration $=0$ & $\mathrm{c}, \mathrm{a}$ & $\mathrm{c}, \mathrm{a}_{\mathrm{a}}$ & $\mathrm{c}, \_\mathrm{a}$ & $\mathrm{d}, \mathrm{a}$ & $\mathrm{d}, \_\mathrm{a}$ & $\_\mathrm{a}$ & $\mathrm{b}$ & & & & & & \\
\hline $\begin{array}{l}\text { iteration }=1 \\
\text { iteration }=2\end{array}$ & & & & & & & $\mathrm{c}, \mathrm{a}$ & $\mathrm{c}, \_\mathrm{a}$ & $\mathrm{c}, \_\mathrm{a}$ & $\mathrm{d}, \mathrm{a}$ & $\mathrm{d}, \_\mathrm{a}$ & a & $\begin{array}{l}\mathrm{b} \\
\mathrm{c}, \mathrm{a}\end{array}$ \\
\hline
\end{tabular}

Table 2 Buffer requirements for the schedule.

\begin{tabular}{|c|c|c|c|c|c|c|c|c|c|c|c|c|c|}
\hline \multirow{3}{*}{ Time } & \multirow{3}{*}{$\begin{array}{l}\text { Actor(s) } \\
\text { Fired }\end{array}$} & \multicolumn{12}{|c|}{ Buffer requirements on arcs } \\
\hline & & \multicolumn{2}{|l|}{$(\mathrm{a}, \mathrm{b})$} & \multicolumn{2}{|l|}{$(b, a)$} & \multicolumn{2}{|l|}{$(b, d)$} & \multicolumn{2}{|l|}{$(\mathrm{d}, \mathrm{b})$} & \multicolumn{2}{|l|}{$(\mathrm{c}, \mathrm{d})$} & \multicolumn{2}{|l|}{$(\mathrm{d}, \mathrm{c})$} \\
\hline & & Before & After & Before & After & Before & After & Before & A & Before & After & Before & After \\
\hline 0 & $\mathrm{c}, \mathrm{a}$ & 0 & 0 & 3 & 3 & 2 & 2 & 1 & 1 & 0 & 2 & 6 & 4 \\
\hline 1 & $\mathrm{c}, \mathrm{a}$ & 0 & 0 & 3 & 3 & 2 & 2 & 1 & 1 & 2 & 4 & 4 & 2 \\
\hline 2 & $\mathrm{c}, \mathrm{a} a$ & 0 & 1 & 3 & 2 & 2 & 2 & 1 & 1 & 4 & 6 & 2 & 0 \\
\hline 3 & $\mathrm{~d}, \mathrm{a}$ & 1 & 1 & 2 & 2 & 2 & 1 & 1 & 2 & 6 & 3 & 0 & 3 \\
\hline 4 & $\mathrm{~d}, \_\mathrm{a}$ & 1 & 1 & 2 & 2 & 1 & 0 & 2 & 3 & 3 & 0 & 3 & 6 \\
\hline 5 & $\mathrm{~b}^{-\mathrm{a}}$ & 1 & 2 & 2 & 1 & 0 & 0 & 3 & 3 & 0 & 0 & 6 & 6 \\
\hline 6 & $\mathrm{c}, \mathrm{a}$ & 2 & 0 & 1 & 3 & 0 & 2 & 3 & 1 & 0 & 2 & 6 & 4 \\
\hline 7 & $\mathrm{c}, \mathrm{a}_{-}$ & 0 & 0 & 3 & 3 & 2 & 2 & 1 & 1 & 2 & 4 & 4 & 2 \\
\hline 8 & $\mathrm{c}, \mathrm{a} \mathrm{a}$ & 0 & 1 & 3 & 2 & 2 & 2 & 1 & 1 & 4 & 6 & 2 & 0 \\
\hline 9 & $\mathrm{~d}, \mathrm{a}$ & 1 & 1 & 2 & 2 & 2 & 1 & 1 & 2 & 6 & 3 & 0 & 3 \\
\hline 10 & $\mathrm{~d}, \_\mathrm{a}$ & 1 & 1 & 2 & 2 & 1 & 0 & 2 & 3 & 3 & 0 & 3 & 6 \\
\hline 11 & $\mathrm{~b}^{-\mathrm{a}}$ & 1 & 2 & 2 & 1 & 0 & 0 & 3 & 3 & 0 & 0 & 6 & 6 \\
\hline 12 & $\mathrm{c}, \mathrm{a} \mathrm{a}$ & 2 & 0 & 1 & 3 & 0 & 2 & 3 & 1 & 0 & 2 & 6 & 4 \\
\hline
\end{tabular}

